\title{
Multiple Primary Tumours, How Frequent we Can Offer Curative Therapy?
}

\author{
Omima Elemam ${ }^{1,2}$, Seham Abdelkhalek ${ }^{1,3}$, Doaa Abdelmoety ${ }^{4}$, Reem Baraka ${ }^{5}$, \\ Mervat Yousef ${ }^{1,6}$
}

${ }^{1}$ Oncology Center, King Abdullah Medical City, Makkah, Saudi Arabia. ${ }^{2}$ Oncology Center, Mansoura University, Mansoura, Egypt. ${ }^{3}$ Radiotherapy Department, Mansoura University, Mansoura, Egypt. ${ }^{4}$ Research Center, King Abdullah Medical City, Makkah, Saudi Arabia. ${ }^{5}$ Department of Biology, Colorado State University, Fort Collins, USA. ${ }^{6}$ Radiotherapy Department, Assiut University, Assiut, Egypt.

\begin{abstract}
Background: Patients with Multiple-primary Malignancies are usually excluded from clinical trials. Clinical information re-distribution, associations, response to treatment and prognosis are scared. Collecting information will help us to expect the impact of prior therapies and to teach us how to best treat them. This study aims to report cases in our society and to see if we have a special predilection of certain Multiple-primary Malignancies in our region based on different geographic and environmental risk factors. Our retrospective study aims to collect these cases and follow their prognosis and treatment response as well as looking for any relation to cancer therapy. Methods: A retrospective study included patients who have two or more histologically diverse primary malignancy, either as synchronous or metachronous malignancy. The study was conducted in King Abdullah Medical City, Saudi Arabia over 7 years period from 2012 to 2019. We collected all patient's clinicopathological information, treatment, and modalities. Results: We collected 53 cases of multiple primary malignancies 26were synchronous (48\%) and 27 were metachronous (52\%). Out of 53 patients, 29 (60\%) were females and $14(40 \%)$ were males. The most common sites for synchronous are breast and endometrial cancer. Curative treatment could be offered in 19 patients (73\%). For metachronous tumours, the most common primary tumour was breast cancer, while the most common second malignancy was colorectal cancer. Curative treatment could be offered in 15 patients (53\%). Conclusion: Multiple primary malignancies represent a small proportion of our cases, with no special predilection in our society. Multiple primary malignancies did not signify a poor prognosis; besides nonmetastatic cases showed a good response to therapy. We should not forget the possibility of a second primary tumour as these cases can be reasonably treated with curative intent.
\end{abstract}

Keywords: Double primary malignancy- Multiple primary malignancies- prognosis- cure rate- cancer risk

Asian Pac J Cancer Care, 5 (2), 71-78

\section{Introduction}

Multiple-primary cancers are defined as primary malignant tumours of different histological origins in a single patient. Recently, there has been an increase in the number of patients diagnosed with multiple-primary cancers; attributed to improved diagnostic techniques and prolonged life span of patients with malignancy. Now we are aware that most multiple primary cancers are double primary cancers [1-2-3].

The incidence of Multiple-Primary Malignancies has
Submission Date: 02/19/2020Ａcceptance Date: 05/03/2020

\footnotetext{
Corresponding Author:

Dr. Omima Elemam

Oncology Center, KAMC, KSA, Makkah, Saudi Arabia. Medical Oncology, Oncology Centre, Mansoura University, Mansoura, Egypt.

Email: omymaelemam@gmail.com
} statistical analyses of Double-Primary Malignancies was carried out by Bugher in 1934, he derived an equation for the probability of death from cancer during a specified period of age with a coincidental Second Malignancy [5]. According to the definition used, the overall reported frequency of Multiple-Primary Cancers ranges from 2\% to $17 \%[6]$.

The criteria used for the diagnosis of Double-Primary 
malignancies has been primarily given by Warren and Gates [7]. While, the two most commonly used definitions were provided by the SEER Program (Surveillance, Epidemiology, and End Results) and the IACR/IARC (International Association of Cancer Registries and International Agency for Research on Cancer) [8]. The SEER database considers single tumours at different sites in the same organ (e.g., colon) as multiple sites. The IACR/IARC rules are more limited; only one tumour is recorded for an organ, regardless of time, unless there are histologic differences. Additionally, The SEER database advocates the use of a 2-month period to distinguish between Synchronous and Metachronous MultiplePrimaries, whereas IARC recommends a 6-month period [9-10].

The theory regarding the origin of the majority of Multiple-Primary cancers is that they arise due to random chance, but different mechanisms have been suggested to be involved in Multiple-Primary Cancers, such as the family history, immunologic, genetic defects, exposure to carcinogens, radiation, chemotherapy, and field cancerization [11].

Germline mutations in mismatch repair genes can produce susceptibility to cancers of the colorectum, ovary, stomach, small bowel, upper uroepithelial tract, hepatobiliary tract, and brain. Li-Fraumeni syndrome (LFS), an autosomal- dominant disorder, features the occurrence of breast cancer in young women and of soft tissue sarcomas, osteosarcomas, brain tumours, acute leukaemia, and adrenocortical tumours in children and young adults [12]. Germline mutations in the p53 tumour suppressor gene (also known as TP53) have been identified in approximately one-half of LFS families in the literature [13] and cigarette smoking that affects the risk of several cancer types.

All the information about double malignancy came from case reports with very few centers reporting their experience and none from our region. We needed to collect our experience in treating those cases. Collecting information will aid us in expecting the impact of prior therapies and teach us how to best treat them.

This is a retrospective study with a single medical facility's experience with Multiple-Primary cancer cases. This study aims to report cases of Double-Primary Malignancy in our society and see if we have a special predilection of certain Double-Primary cancers based on different geographic and environmental risk factors.

\section{Materials and Methods}

This was a retrospective observational study carried out at King Abdullah Medical City, Makkah, Saudi Arabia, from January 2012 to December 2019. All Patients with malignant tumors of different histological origins were defines as histologically confirmed Multiple-Primary Malignancy cases. Patients were identified through a retrospective review of medical records excluding patients with insufficient histopathological data.

The data collected were the patients' characteristics, pathological data, and outcome information. Moreover, this research protocol was approved by the Institutional Review Board Committee. Since the study performed is retrospective, we obtained a waiver of informed consent from IRB.

\section{Results}

We collected 53 cases of Multiple-Primary malignancies 26 were synchronous $(48 \%)$ and 27 were metachronous $(52 \%)$. Out of 53 patients, $29(60 \%)$ were females and $14(40 \%)$ were males.

The most common sites for synchronous, Table 1, are breast and endometrial cancer. Metastasis at diagnosis was present in 7 patients (23\%). The median age of diagnosis for synchronous tumours was 61 years (range: 27 to 83 years), 4 patients were male (26\%). CT scan for staging workup resulted in the detection of a second tumour in 21 patients 5 patients of which was diagnosed during pathological examination. Curative treatment could be offered in 19 patients $(73 \%)$.

For metachronous tumours, Table 2, the median age of diagnosis for the second primary neoplasm was 54years (range: 34 to 82 years) 3 patients of which were male. The median interval of six years was observed, the most common sites of a primary tumour were breast, the most common second malignancy was colorectal cancer and Metastasis at diagnosis were present in 7 patients $(25 \%)$. Curative treatment could be offered in 15 patients (53\%).

\section{Discussion}

Multiple primaries [14] are more than one tumour arising in different sites and or of different histology either synchronous or metachronous depends on the duration between them, 2-month according to SEER data [15] and 6 months according to IARC. In our study, we used the definition of IARC. The burden of multiple tumours is expected to increase due to the use of accurate imaging techniques. In a single facility in Saudi Arabia, we collected 54 cases over 7 years. Multiple-Primary did not always signify a bad prognosis as we treated all non-metastatic cases with curative intent.

It is most imperative to diagnose it early before the patient reaches the metastatic stage. This means that we should have a high degree of suspicion. The role of the radiologist is crucial as usually, radiologists are first to flag for suspicion of multiple tumours. In our study, most of the synchronous tumours were detected initially by CT scan then confirmed pathologically. Examples from our study as $\mathrm{CT}$ scan done for a patient with ovarian cancer showed a breast mass or CT scan showed a speculated lung lesion in a patient with breast cancer or showed renal mass in a patient with nasopharyngeal cancer.

The response to therapy is always an alarm for the physician to review his pathology by repeating the biopsy of the metastatic disease. In our study a patient with breast cancer who developed lung nodules treated treated with chemotherapy as. Metastatic breast then the poor response to chemotherapy urged us to biopsy the largest metastatic nodule and to our surprise came to be second primary 
Table 1. Patients with Synchronous Advanced Multiple Primary Tumours

\begin{tabular}{|c|c|c|c|c|c|c|c|c|}
\hline $\begin{array}{l}\text { Age At } \\
\text { diagnosis }\end{array}$ & sex & Primary & Secondary & $\begin{array}{l}\text { Detected } \\
\text { by }\end{array}$ & Treatment & $\begin{array}{l}\text { Metastasis } \\
\text { at } \\
\text { presentation }\end{array}$ & $\begin{array}{l}\text { State of last } \\
\text { follow up }\end{array}$ & $\begin{array}{l}\text { Outcome /duration } \\
\text { from diagnosis to } \\
\text { death }\end{array}$ \\
\hline 79 & $\mathrm{~F}$ & $\begin{array}{l}\text { Stage II breast } \\
\text { cancer } \\
\text { invasive } \\
\text { ductal cancer (IDC) }\end{array}$ & $\begin{array}{l}\text { Stage IV ovarian } \\
\text { serous cancer }\end{array}$ & CT scan & $\begin{array}{l}\text { Mastectomy } \\
\text { and chemotherapy } \\
\text { carboplatin/ } \\
\text { Paclitaxel }\end{array}$ & yes & dead & $\begin{array}{l}\text { Refused surgery } \\
\text { for the ovary then } \\
\text { received paclitaxel } \\
\text { weekly then } \\
\text { palliative care }\end{array}$ \\
\hline 68 & $\mathrm{~F}$ & $\begin{array}{l}\text { Right breast IDC } \\
11 / 2017\end{array}$ & $\begin{array}{l}\text { Stage IIIC high } \\
\text { grade serous cancer }\end{array}$ & CT scan & $\begin{array}{l}\text { TAH \&BSO } \\
\text { Omentectomy. } \\
\text { Adjuvant Carbo/ } \\
\text { paclitaxel } \\
\text { No surgery for breast }\end{array}$ & No & Alive & Under follow up \\
\hline 61 & $\mathrm{~F}$ & $\begin{array}{l}\text { Right breast IDC } \\
\text { stage IIIB }\end{array}$ & $\begin{array}{l}\text { Stage IIA colon } \\
\text { cancer }\end{array}$ & CT scan & $\begin{array}{l}\text { BCS ALND } \\
\text { Chemotherapy FEC/ } \\
\text { Docetaxel } \\
\text { Sigmoidectomy } \\
\text { letrozole }\end{array}$ & No & free & Under follow up \\
\hline 44 & $\mathrm{~F}$ & $\begin{array}{l}\text { Right breast cancer } \\
\text { IDC }\end{array}$ & $\begin{array}{l}\text { Appendicular } \\
\text { mucinous } \\
\text { adenocarcinoma } \\
\text { stage IV }\end{array}$ & CT scan & MRM tamoxifen & yes & dead & 5 months \\
\hline 52 & $\mathrm{~F}$ & $\begin{array}{l}\text { Left breast stage } \\
\text { IIIA IDC }\end{array}$ & $\begin{array}{l}\text { Stage IA } \\
\text { endometrioid } \\
\text { adenocarcinoma of } \\
\text { the uterus }\end{array}$ & CT scan & $\begin{array}{l}\text { TAH\& BSO o } \\
\text { mentectomy PLND } \\
\text { Left MRM } \\
\text { Chemotherapy } \\
\text { FEC/docetaxel } \\
\text { Radiotherapy } \\
\text { Letrozole }\end{array}$ & No & free & $\begin{array}{l}\text { Positive family } \\
\text { history of ovarian } \\
\text { cancer BRCA } \\
\text { mutation positive } \\
\text { Under follow-up }\end{array}$ \\
\hline 63 & $\mathrm{~F}$ & Breast IDC & $\begin{array}{l}\text { Endometrial } \\
\text { endometrioid } \\
\text { adenocarcinoma }\end{array}$ & CT scan & $\begin{array}{l}\text { Neoadjuvant with } \\
\text { dual anti-HER } 2 \\
\text { therapy. Followed by } \\
\text { surgery for both }\end{array}$ & No & Alive & $\begin{array}{l}\text { The patent currently } \\
\text { under adjuvant } \\
\text { therapy }\end{array}$ \\
\hline 63 & $\mathrm{~F}$ & $\begin{array}{l}\text { Triple negative left } \\
\text { breast IDC }\end{array}$ & $\begin{array}{l}\text { Hormone receptor } \\
\text { positive right breast } \\
\text { cancer }\end{array}$ & $\begin{array}{l}\text { Path } \\
\text { exam }\end{array}$ & $\begin{array}{l}\text { Neoadjuvant AC/ } \\
\text { docetaxel Bilateral } \\
\text { MRM }\end{array}$ & No & Alive & $\begin{array}{l}\text { Currently } \\
\text { under adjuvant } \\
\text { hormonal therapy }\end{array}$ \\
\hline 65 & $\mathrm{~F}$ & Right breast IDC & $\begin{array}{l}\text { Stage IV Rectal } \\
\text { adenocarcinoma }\end{array}$ & CT scan & $\begin{array}{l}\text { Right MRM } \\
\text { Chemotherapy } \\
\text { for rectal cancer }\end{array}$ & yes & Yes liver & $\begin{array}{l}\text { Still under } \\
\text { chemotherapy }\end{array}$ \\
\hline 75 & $\mathrm{~F}$ & $\begin{array}{l}\text { Stage IVB } \\
\text { endometrioid } \\
\text { adenocarcinoma }\end{array}$ & $\begin{array}{l}\text { Stage I mucinous } \\
\text { carcinoma of the } \\
\text { breast }\end{array}$ & CT scan & $\begin{array}{l}\text { Carboplatin } \\
\text { paclitaxel. But } \\
\text { Refused surgery }\end{array}$ & yes & Dead & $\begin{array}{l}\text { Refused surgery for } \\
\text { the endometrium } \\
\text { after very good } \\
\text { response to } \\
\text { chemotherapy }\end{array}$ \\
\hline 49 & $\mathrm{~F}$ & $\begin{array}{l}\text { Stage IA } \\
\text { endometrial } \\
\text { endometrioid } \\
\text { adenocarcinoma }\end{array}$ & $\begin{array}{l}\text { Stage IA mucinous } \\
\text { borderline tumour }\end{array}$ & $\begin{array}{l}\text { Path } \\
\text { exam }\end{array}$ & $\begin{array}{l}\text { TAH \&BSO } \\
\text { infracolic } \\
\text { omentectomy }\end{array}$ & No & free & Under follow up \\
\hline 36 & $\mathrm{~F}$ & $\begin{array}{l}\text { Stage IA } \\
\text { endometrial } \\
\text { adenosequamus } \\
\text { cancer }\end{array}$ & $\begin{array}{l}\text { Papillary thyroid } \\
\text { cancer }\end{array}$ & CT scan & $\begin{array}{l}\text { TAH \&BSO } \\
\text { Total thyroidectomy }\end{array}$ & No & free & Underfollow up \\
\hline $64 *$ & $\mathrm{~F}$ & $\begin{array}{l}\text { Stage IA } \\
\text { endometrial } \\
\text { endometrioid } \\
\text { adenocarcinoma } \\
\text { NSCLC } \\
\text { stage T1bN0 }\end{array}$ & Cancer rectum & CT scan & $\begin{array}{l}\text { TAH \&BSO } \\
\text { Omentectomy+ } \\
\text { Choleycystectomy } \\
\text { Right lung lobectomy } \\
\text { and hilar lymph } \\
\text { node excision LAR }\end{array}$ & No & free & Under follow up \\
\hline 56 & $\mathrm{~F}$ & $\begin{array}{l}\text { Stage I Uterine } \\
\text { leiomyosarcoma }\end{array}$ & $\begin{array}{l}\text { Stage IA Ovarian } \\
\text { endometrioid } \\
\text { borderline tumour }\end{array}$ & $\begin{array}{l}\text { Path } \\
\text { exam }\end{array}$ & $\begin{array}{l}\text { TAH \&BSO } \\
\text { Omentectomy } 7 / 2018\end{array}$ & No & alive & Under follow up \\
\hline 72 & $\mathrm{~F}$ & $\begin{array}{l}\text { Stage IA } \\
\text { carcinosarcoma of } \\
\text { the uterus }\end{array}$ & $\begin{array}{l}\text { Stage I mucinous } \\
\text { carcinoma of the } \\
\text { breast }\end{array}$ & CT scan & $\begin{array}{l}\text { TAH \&BSO } \\
\text { Followed by Adjuvant } \\
\text { carboplatin/paclitaxel, } \\
\text { Radiotherapy } \\
\text { External pelvic } \\
\text { Right MRM } \\
\text { Adjuvant Letrozole }\end{array}$ & No & free & $\begin{array}{l}\text { Relapsed } 6 \text { years } \\
\text { later with metastatic } \\
\text { carcinosarcoma } \\
\text { shifted to palliative } \\
\text { care after two cycles } \\
\text { of chemotherapy }\end{array}$ \\
\hline
\end{tabular}


Continued Table 1.

\begin{tabular}{|c|c|c|c|c|c|c|c|c|}
\hline $\begin{array}{l}\text { Age At } \\
\text { diagnosis }\end{array}$ & Sex & Primary & Secondary & $\begin{array}{l}\text { Detected } \\
\text { by }\end{array}$ & Treatment & $\begin{array}{l}\text { Metastasis } \\
\text { at } \\
\text { presentation }\end{array}$ & $\begin{array}{l}\text { State of last } \\
\text { follow up }\end{array}$ & $\begin{array}{l}\text { Outcome /duration } \\
\text { from diagnosis to } \\
\text { death }\end{array}$ \\
\hline 27 & $\mathrm{~F}$ & $\begin{array}{l}\text { Left foot } \\
\text { leiomyosarcoma }\end{array}$ & thymoma & CT scan & Surgery for both & No & free & Under follow up \\
\hline 39 & $\mathrm{~F}$ & $\begin{array}{l}\text { Low grade brain } \\
\text { glioma large } \\
\text { infiltrative mass }\end{array}$ & $\begin{array}{l}\text { Abdominal } \\
\text { mass GIST }\end{array}$ & CT scan & Bilateral V/P shunt & No & dead & 21 months \\
\hline 78 & M & $\begin{array}{l}\text { Stage IV } \\
\text { nasopharyngeal } \\
\text { cancer } \\
\text { undifferentiated }\end{array}$ & $\begin{array}{l}\text { Hepatocellular } \\
\text { carcinoma } \\
\text { Cirrhosis, LCF }\end{array}$ & CT scan & $\begin{array}{l}\text { Radiotherapy } \\
\text { incomplete course }\end{array}$ & yes & dead & 6 months \\
\hline 47 & $\mathrm{~F}$ & $\begin{array}{l}\text { Colon cancer high } \\
\text { grade } \\
\text { adenocarcinoma }\end{array}$ & $\begin{array}{l}\text { Hodgkin's disease } \\
\text { classical type stage } \\
\text { IV }\end{array}$ & CT scan & ABVD & yes & dead & 9 months \\
\hline 83 & M & $\begin{array}{l}\text { Rectal moderately } \\
\text { differentiated } \\
\text { cancer }\end{array}$ & $\begin{array}{l}\text { Metastatic Prostate } \\
\text { cancer GS } 4+4\end{array}$ & CT scan & $\begin{array}{l}\text { Goserelin / } \\
\text { bicalutamide }\end{array}$ & yes & dead & 8 months \\
\hline 69 & M & $\begin{array}{l}\text { Sigmoid } \\
\text { adenocarcinoma } \\
\text { T2N1 }\end{array}$ & $\begin{array}{l}\text { stageIIB Lung } \\
\text { adenocarcinoma } \\
\text { Lung carcinoid } \\
\text { tumour low grade }\end{array}$ & CT scan & $\begin{array}{l}\text { Surgery for both then } \\
\text { adjuvant } \\
\text { chemotherapy } \\
\text { for lung carboplatin/ } \\
\text { pemetrexed }\end{array}$ & no & Alive & Under follow up \\
\hline 60 & $\mathrm{~F}$ & $\begin{array}{l}\text { Gastric } \\
\text { adenocarcinoma } \\
\text { T4N3M0 }\end{array}$ & $\begin{array}{l}\text { Lung mucinous } \\
\text { adenocarcinoma }\end{array}$ & CT scan & $\begin{array}{l}\text { Neoadjuvant } \\
\text { chemotherapy ECF } \\
\text { for gastric cancer, } \\
\text { Gastrectomy then } \\
\text { Lung lobectomy }\end{array}$ & No & Dead & 13 months \\
\hline 78 & M & $\begin{array}{l}\text { Prostate } \\
\text { adenocarcinoma }\end{array}$ & $\begin{array}{l}\text { Pleomorphic } \\
\text { undifferentiated } \\
\text { Sarcoma left femur }\end{array}$ & CT scan & $\begin{array}{l}\text { Orchiectomy } \\
\text { radiotherapy }\end{array}$ & no & Alive & Lost follow up \\
\hline 59 & M & $\begin{array}{l}\text { Renal cell } \\
\text { carcinoma }\end{array}$ & $\begin{array}{l}\text { Metastatic } \\
\text { nasopharyngeal } \\
\text { cancer }\end{array}$ & CT scan & $\begin{array}{l}\text { Target therapy } \\
\text { plus chemotherapy }\end{array}$ & yes & Alive & $\begin{array}{l}\text { localized RCC } \\
\text { refused surgery } \\
\text { received pazopanib } \\
\text { changed to sunitinib } \\
\text { metastatic NPC } \\
\text { received gemcitabine } \\
10 \text { cycles then start } \\
2 \text { nd line } \\
\text { docetaxel }\end{array}$ \\
\hline 68 & $\mathrm{~F}$ & $\begin{array}{l}\text { Stage I gastric } \\
\text { leiomyosarcoma }\end{array}$ & $\begin{array}{l}\text { Stage I ovarian } \\
\text { serous cancer }\end{array}$ & CT scan & $\begin{array}{l}\text { Partial gastrectomy, } \\
\text { ovarian cystectomy } \\
\text { then Neoadjuvant } \\
\text { carboplatin/paclitaxel } \\
\text { then debulking } \\
\text { surgery }\end{array}$ & no & free & Underfollow up \\
\hline 34 & $\mathrm{~F}$ & $\begin{array}{l}\text { stage IA high grade } \\
\text { ovarian mucinous } \\
\text { cancer }\end{array}$ & $\begin{array}{l}\text { Stage IA } \\
\text { endometrioid } \\
\text { adenocarcinoma }\end{array}$ & $\begin{array}{l}\text { Path } \\
\text { exam }\end{array}$ & $\begin{array}{l}\text { TAH\&BSO and } \\
\text { omentectomy. } \\
\text { Chemotherapy } \\
\text { carboplatin/paclitaxel }\end{array}$ & no & free & Under follow up \\
\hline 45 & $\mathrm{~F}$ & $\begin{array}{l}\text { Stage IC mucinous } \\
\text { ovarian cancer }\end{array}$ & $\begin{array}{l}\text { Stage IB } \\
\text { endometrioid } \\
\text { adenocarcinoma }\end{array}$ & $\begin{array}{l}\text { Path } \\
\text { exam }\end{array}$ & $\begin{array}{l}\text { TAH\&BSO and } \\
\text { omentectomy. } \\
\text { Chemotherapy } \\
\text { carboplatin/paclitaxel } \\
\text { Followed by } \\
\text { radiotherapy }\end{array}$ & no & free & Under follow up \\
\hline
\end{tabular}

\section{Lung Cancer.}

Cancer patients who survive their primary tumour always have a high risk to develop a second primary and this is due to many reasons like genetic predisposition as one of our patients who has Double Synchronous Primary Breast Cancer and Endometrial Cancer gave a strong family history of ovarian cancer and her BRCA genetic testing came to be positive.

Cancer treatment is carcinogenic. We are reporting leukaemia in ovarian cancer patients treated with chemotherapy, breast cancer patients treated for DLBCL, and breast and thyroid cancer in Hodgkin's lymphoma patients treated at a young age. 
For patients with breast cancer, the incidence of second primaries studied and has been reported to range from $4.1 \%$ to $16.4 \%$ [15-16]. An excess risk of endometrial cancer is reported with the use of Tamoxifen [17] Genetic factors as BRCA1 BRCA2 mutations are well-known risk factors for Multiple-Primary [18]. In this study, we reported 7 cases of the Synchronous Second-Primary with breast cancer, 3 cases with ovarian cancer. Also, we reported endometrial cancer in patients with hormone receptor-positive breast cancer with BRCA mutation. AML can be triggered during the first 2 years after radiation therapy and it is also a late effect of chemotherapy. For metachronous tumour in patients with breast cancer, We reported two cases of AML which may be chemotherapy related.

Patients with prostate cancer who received external beam radiotherapy are at increased risk of bladder cancer, rectal cancer and sarcomas within the radiation field after being disease-free for at least 5 years [19]. Second primaries can also occur in patients with prostate cancer owing to genetic factors, especially BRCA mutation [20]. In our study, we reported prostate cancer, rectal cancer, and prostate cancer and sarcoma synchronously.

The most important cause of mortality in Hodgkin's lymphoma is a Second-Primary cancer [21]. We reported a case of colon cancer synchronously with Hodgkin's lymphoma and thyroid cancer. Also, breast cancer that occurred 12 years after ABVD for Hodgkin's lymphoma.

Smoking is an important risk factor not only for lung cancer but also for a Second-Primary Cancer. A 7.9\%of lung cancer cases who acquire a second primary have

Table 2. Patients with Metachronous Advanced Multiple Primary Tumours

\begin{tabular}{|c|c|c|c|c|c|c|c|c|}
\hline Age & Sex & Primary & Treatment & Secondary & Treatment & $\begin{array}{l}\text { Metastasis } \\
\text { at presentation }\end{array}$ & $\begin{array}{l}\text { Interval between } \\
\text { primary and } \\
\text { secondary }\end{array}$ & Recurrence \\
\hline 53 & $\mathrm{~F}$ & $\begin{array}{l}\text { Stage I Breast } \\
\text { IDC }\end{array}$ & $\begin{array}{l}\text { Surgery hormonal } \\
\text { letrozole }\end{array}$ & $\begin{array}{l}\text { Stage II } \\
\text { Colon cancer }\end{array}$ & Surgery, FOLFOX & No & $7 y$ & \\
\hline 50 & F & $\begin{array}{l}\text { Stage III Breast } \\
\text { IDC }\end{array}$ & $\begin{array}{l}\text { Neoadjuvant } \\
\text { chemotherapy } \\
\text { EC/T Surgery, } \\
\text { radiotherapy }\end{array}$ & $\begin{array}{l}\text { Colon } \\
\text { cancer T4N1M0 }\end{array}$ & $\begin{array}{l}\text { SUREGERY } \\
\text { Capecitabine / } \\
\text { Oxaliplatin }\end{array}$ & NO & $14 \mathrm{Y}$ & $\begin{array}{l}\text { Colon cancer } \\
\text { recurrence and } \\
\text { received } \\
\text { chemotherapy } \\
\text { HIPEC and on } \\
\text { chemotherapy }\end{array}$ \\
\hline 64 & $\mathrm{~F}$ & $\begin{array}{l}\text { Stage II triple } \\
\text { negative breast } \\
\text { IDC }\end{array}$ & $\begin{array}{l}\text { Surgery } \\
\text { chemotherapy } \\
\text { radiotherapy }\end{array}$ & $\begin{array}{l}\text { Stage IV } \\
\text { pancreatic } \\
\text { cancer }\end{array}$ & Palliative care & yes & $6 y$ & $\begin{array}{l}\text { Died after } 3 \\
\text { months }\end{array}$ \\
\hline 39 & $\mathrm{~F}$ & $\begin{array}{l}\text { Breast IDC } \\
\text { stage II }\end{array}$ & $\begin{array}{l}\text { Surgery FAC } \\
\text { Tamoxifen }\end{array}$ & Endometrium & $\begin{array}{l}\text { Surgery } \\
\text { radiotherapy }\end{array}$ & no & $11 \mathrm{y}$ & \\
\hline 44 & F & $\begin{array}{l}\text { Stage II breast } \\
\text { IDC }\end{array}$ & $\begin{array}{l}\text { Surgery TEC } \\
\text { radiotherapy } \\
\text { Tam /letrozole }\end{array}$ & $\begin{array}{l}\text { Stage III Uterine } \\
\text { carcinosarcoma }\end{array}$ & $\begin{array}{l}\text { surgery } \\
\text { carbo/paclitaxel }\end{array}$ & & $6 y$ & \\
\hline 42 & F & $\begin{array}{l}\text { STAGE IIA } \\
\text { BREAST IDC }\end{array}$ & $\begin{array}{l}\text { RT MRM } \\
\text { TEC } \\
\text { radiotherapy } \\
\text { Tamoxifen }\end{array}$ & APL M5 & ATRA & no & $2 y$ & $\begin{array}{l}\text { In CR } \\
\text { Under follow up on } \\
\text { Tamoxifen }\end{array}$ \\
\hline 35 & $\mathrm{~F}$ & $\begin{array}{l}\text { Stage II breast } \\
\text { IDC }\end{array}$ & $\begin{array}{l}\text { Surgery FEC/ } \\
\text { Docetaxel } \\
\text { Radiotherapy } \\
\text { tamoxifen }\end{array}$ & AML & FLAG then IDC. & no & $5 y$ & $\begin{array}{l}\text { In CR } \\
\text { Under FU }\end{array}$ \\
\hline 51 & F & $\begin{array}{l}\text { Stage IIIA } \\
\text { Breast cancer } \\
\text { IDC Her2neu } \\
\text { positive disease }\end{array}$ & $\begin{array}{l}\text { Surgery } \\
\text { chemotherapy } \\
\text { Radiotherapy }\end{array}$ & $\begin{array}{l}\text { Lung squamous } \\
\text { cell lung cancer }\end{array}$ & Chemotherapy & no & $2 y$ & $\begin{array}{l}\text { Still under } \\
\text { chemotherapy }\end{array}$ \\
\hline 47 & F & $\begin{array}{l}\text { ER+ breast } \\
\text { cancer }\end{array}$ & $\begin{array}{l}\text { Surgery and } \\
\text { adjuvant chemo. } \\
\text { Hormonal and } \\
\text { radiotherapy }\end{array}$ & $\begin{array}{l}\text { Stage IIA } \\
\text { triple negative } \\
\text { breast cancer }\end{array}$ & $\begin{array}{l}\text { Surgery } \\
\text { and chemotherapy }\end{array}$ & Yes & $8 \mathrm{y}$ & Under follow up \\
\hline 45 & $\mathrm{~F}$ & $\begin{array}{l}\text { Papillary } \\
\text { thyroid cancer }\end{array}$ & $\begin{array}{l}\text { Surgery total } \\
\text { thyroidectomy, } \\
\text { radioactive iodine }\end{array}$ & $\begin{array}{l}\text { Stage III } \\
\text { Follicular } \\
\text { lymphoma }\end{array}$ & FCR & & $3 y$ & Under follow up \\
\hline 41 & $\mathrm{~F}$ & $\begin{array}{l}\text { Papillary } \\
\text { Thyroid Cancer }\end{array}$ & $\begin{array}{l}\text { Surgery } \\
\text { radiotherapy }\end{array}$ & $\begin{array}{l}\text { Stage IC Ovarian } \\
\text { serous cancer }\end{array}$ & $\begin{array}{l}\text { Debulking surgery } \\
\text { Adjuvant } \\
\text { chemotherapy }\end{array}$ & no & $9 y$ & dead \\
\hline 72 & M & $\begin{array}{l}\text { Papillary } \\
\text { Thyroid cancer }\end{array}$ & $\begin{array}{l}\text { Thyroid surgery } \\
\text { and ablation on } \\
\text { replacement }\end{array}$ & Stage IV NSCLC & $\begin{array}{l}\text { Chemotherapy } \\
\text { and palliative } \\
\text { radiotherapy }\end{array}$ & yes & $20 \mathrm{y}$ & dead \\
\hline 59 & $\mathrm{~F}$ & $\begin{array}{l}\text { CML chronic } \\
\text { phase }\end{array}$ & $\begin{array}{l}\text { TKI Imatinib, } \\
\text { desatinib }\end{array}$ & Colon cancer & $\begin{array}{l}\text { Chemotherapy. } \\
\text { Radiotherapy }\end{array}$ & & $9 y$ & Dead \\
\hline
\end{tabular}


Continued Table 2 .

\begin{tabular}{|c|c|c|c|c|c|c|c|c|}
\hline Age & Sex & Primary & Treatment & Secondary & Treatment & $\begin{array}{l}\text { Metastasis } \\
\text { at presentation }\end{array}$ & $\begin{array}{l}\text { Interval between } \\
\text { primary and } \\
\text { secondary }\end{array}$ & Recurrence \\
\hline 35 & $\mathrm{~F}$ & CML & TKI & $\begin{array}{l}\text { Tracheal } \\
\text { adenocarcinoma }\end{array}$ & $\begin{array}{l}\text { Surgery } \\
\text { radiotherapy }\end{array}$ & no & $15 \mathrm{y}$ & $\begin{array}{l}\text { In MMR } \\
\text { Under follow up }\end{array}$ \\
\hline 70 & $\mathrm{~F}$ & Colon cancer & $\begin{array}{l}\text { Surgery, } \\
\text { XELOX }\end{array}$ & Breast & $\begin{array}{l}\text { Docetaxel } \\
\text { trastuzumab }\end{array}$ & yes & $5 y$ & dead \\
\hline $68^{*}$ & $\mathrm{~F}$ & $\begin{array}{l}\text { Stage II } \\
\text { adenocarcinoma } \\
\text { Colon cancer }\end{array}$ & $\begin{array}{l}\text { Surgery } \\
\text { radiotherapy } \\
\text { chemotherapy }\end{array}$ & $\begin{array}{l}\text { CML } \\
\text { Then developed } \\
\text { thyroid cancer }\end{array}$ & $\begin{array}{l}\text { TKI } \\
\text { Chemotherapy for } \\
\text { colon }\end{array}$ & No & $2 y$ & dead \\
\hline 80 & $\mathrm{~F}$ & $\mathrm{HCC}$ & $\begin{array}{l}\text { HA } \\
\text { chemoembolization }\end{array}$ & $\begin{array}{l}\text { Breast cancer } \\
\text { stage IV liver } \\
\text { bone Mets }\end{array}$ & $\begin{array}{l}\text { Trastuzumab } \\
\text { Hormonal } \\
\text { treatment }\end{array}$ & yes & $2 y$ & dead \\
\hline 70 & $\mathrm{~F}$ & $\begin{array}{l}\text { Stage IV } \\
\text { adenocarcinoma } \\
\text { of the gall } \\
\text { bladder }\end{array}$ & $\begin{array}{l}\text { Surgery then } \\
\text { gemcitabine }\end{array}$ & $\begin{array}{l}\text { Stage colon } \\
\text { cancer }\end{array}$ & $\begin{array}{l}\text { Surgery } \\
\text { chemotherapy } \\
\text { FOLFOX / } \\
\text { bevacizumab }\end{array}$ & yes & $1 y$ & dead \\
\hline 66 & $\mathrm{~F}$ & $\begin{array}{l}\text { Stage } \\
\text { endometrial } \\
\text { endometrioid } \\
\text { adenocarcinoma }\end{array}$ & Surgery & Breast DCIS & $\begin{array}{l}\text { Surgery } \\
\text { Tamoxifen }\end{array}$ & No & $3 y$ & free \\
\hline 41 & $\mathrm{~F}$ & $\begin{array}{l}\text { Stage II } \\
\text { granulosa cell } \\
\text { tumour of the } \\
\text { ovary }\end{array}$ & $\begin{array}{l}\text { Surgery } \\
\text { chemotherapy } \\
\text { VAC }\end{array}$ & $\begin{array}{l}\text { Stage IV } \\
\text { Carcinoid } \\
\text { tumour of the } \\
\text { pancreas }\end{array}$ & octreotide & yes & $10 \mathrm{y}$ & Under octreotide \\
\hline 82 & $\mathrm{~F}$ & $\begin{array}{l}\text { Stage IA } \\
\text { endometrial } \\
\text { endometrioid } \\
\text { Adenocarcinoma }\end{array}$ & Surgery & $\begin{array}{l}\text { Stage I breast } \\
\text { IDC }\end{array}$ & $\begin{array}{l}\text { Hormonal and } \\
\text { radiotherapy }\end{array}$ & no & $1 \mathrm{y}$ & free \\
\hline 65 & $\mathrm{~F}$ & $\begin{array}{l}\text { Stage IIIc serous } \\
\text { ovarian cancer }\end{array}$ & $\begin{array}{l}\text { Neoadjuvant } \\
\text { chemotherapy } \\
\text { carboplatin / } \\
\text { paclitaxel } \\
\text { Interval debuing } \\
\text { Adjuvant } \\
\text { chemotherapy }\end{array}$ & $\begin{array}{l}\text { Stage IV high } \\
\text { grade } \\
\text { neuroendocrine } \\
\text { tumour }\end{array}$ & $\begin{array}{l}\text { Refused } \\
\text { chemotherapy }\end{array}$ & No & $1 y$ & dead \\
\hline 59 & M & $\begin{array}{l}\text { Cancer larynx } \\
\text { T1N0M0 }\end{array}$ & $\begin{array}{l}\text { Radical } \\
\text { Radiotherapy }\end{array}$ & $\begin{array}{l}\text { Stage IV gastric } \\
\text { cancer }\end{array}$ & $\begin{array}{l}\text { Palliative } \\
\text { chemotherapy and } \\
\text { radiotherapy }\end{array}$ & Yes, liver Mets & $2 y$ & dead \\
\hline 64 & M & $\begin{array}{l}\text { DLBCL stage } \\
\text { IV B }\end{array}$ & Chemotherapy & $\begin{array}{l}\text { Stage IIIB } \\
\text { Mesothelioma }\end{array}$ & Chemotherapy & No & $4 y$ & $\begin{array}{l}\text { Under } \\
\text { Chemotherapy }\end{array}$ \\
\hline $34^{*}$ & $\mathrm{~F}$ & $\begin{array}{l}\text { Stage III B } \\
\text { Hodgkin's } \\
\text { lymphoma }\end{array}$ & ABVD & $\begin{array}{l}\text { Papillary thyroid } \\
\text { cancer And left } \\
\text { breast cancer }\end{array}$ & $\begin{array}{l}\text { Surgery } \\
\text { radioactive iodine }\end{array}$ & No & $12 \mathrm{y}$ & Under follow up \\
\hline $55^{*}$ & $\mathrm{~F}$ & $\begin{array}{l}\text { Stage IIA } \\
\text { DLBCL }\end{array}$ & $\begin{array}{l}\text { Chemotherapy } \\
\text { and radiotherapy }\end{array}$ & $\begin{array}{l}\text { Breast cancer } \\
\text { stage and } \\
\text { follicular } \\
\text { lymphoma stage } \\
\text { IA }\end{array}$ & $\begin{array}{l}\text { Lumpectomy and } \\
\text { ALND } \\
\text { Chemotherapy } \\
\text { Hormonal therapy } \\
\text { for the breast } \\
\text { Radiotherapy for } \\
\text { FL }\end{array}$ & No & $2 y$ & Under follow up \\
\hline 54 & $\mathrm{~F}$ & $\begin{array}{l}\text { Stage IIIA NHL } \\
\text { follicular GII }\end{array}$ & $\begin{array}{l}\text { Rituximab for } 4 \\
\text { weeks and then } \\
\text { maintenance }\end{array}$ & $\begin{array}{l}\text { Hodgkin's } \\
\text { lymphoma in } \\
\text { axillary lymph } \\
\text { node }\end{array}$ & AVD & No & $2 y$ & Under follow up \\
\hline
\end{tabular}

*Triple malignancies; IDC, Invasive ductal carcinoma; DLBCL, diffuse large b cell lymphoma; TAH BSO, total abdominal hysterectomy and bilateral salpingoophrectomy; BCS ALND, breast conserving surgery and axillary lymph node dissection; MRM, modified radical mastectomy NSCLC non-small cell lung cancer; ABVD, Adriamycin, bleomycin vinblastine dacarbazine; AML, Acute myeloid leukaemia; APL, Acute promyelocytic leukaemia. 
SCLC [22]. In this study, we reported colon cancer 1 year after lung cancer and gastroesophageal cancer that was diagnosed 18 months after the lung cancer diagnosis.

The treatment decision of synchronous tumours is not straightforward and usually requires a multidisciplinary approach, one of our patients had a synchronous breast and endometrial cancer. We discussed the case in our tumour board, and we decided to give her neoadjuvant chemotherapy followed by surgery MRM and TAH\&BSO in the operating room by two surgeons.

We treated our patients with curative intent in more than $60 \%$ of cases so, we should always be aware of the possibility of a second primary cancer. Late metastatic spread in a patient with triple-negative breast cancer led us to suspect second primary and diagnose pancreatic cancer. Also, low tumour marker in ovarian cancer patient which was initially high was found to have a second primary neuroendocrine tumour, continued smoking history should alert us about this possible important carcinogen.

We should inform our patients about the late side effects of their treatment, particularly Second-Primary Malignancies, by including it in the consent form. Such actions would educate patients on the value of continuous surveillance and avoiding all possible carcinogens especially smoking in addition to encouraging them for a healthy lifestyle [23-24].

Patients with multiple primaries are usually excluded from clinical trials and there are no established guidelines to treat these cases. we need clinical trials to study the new histology non-specific medications like (immunotherapy, biologic therapy. etc)

Finally, in our medical facility, we adopted the policy of referring our cases with Multiple-Primary to our genetic oncology clinic for evaluation and genetic testing; this hopefully will help us gain more knowledge about patients with hereditary cancer. we will report these data separately.

In conclusion, we are expecting an increase in the prevalence of Multiple-Primary tumours due to increased accuracy of diagnostic techniques in addition to novel target therapy that may increase the risk. Hereditary cancer syndrome, smoking, cancer therapy are all risk factors. we need to pick these cases as early as possible before the development of metastasis as this has a marked impact on patient survival. Treatment decisions for these cases should be based on a multidisciplinary approach. Research on this topic is an unmet need particularly the genetic background for developing second primary cancers. To reflect more of a real-life population, we need clinical trials investigating those patients in detail to increase the physician's awareness that these cases are not rare, and they need to be treated with curative intent in most situations.

\section{References}

1. Siegel R, Miller K, Jemal A. Cancer statistics, 2019. CA: A Cancer Journal for Clinicians. 2019;69(1):7-34.

2. Noh S, Yoon J, Ryoo U, Choi C, Sung C, Kim T et al. A case report of quadruple cancer in a single patient including the breast, rectum, ovary, and endometrium. Journal of Gynecologic Oncology. 2008;19(4):265.
3. Lee J, Moon W, Park S, Park M, Kim K, Jang L et al. Triple Synchronous Primary Cancers of Rectum, Thyroid, and Uterine Cervix Detected during the Workup for Hematochezia. Internal Medicine. 2010;49(16):1745-1747.

4. Owen L. MULTIPLE MALIGNANT NEOPLASMS. JAMA: The Journal of the American Medical Association. 1921;76(20):1329

5. Bugher JC. The probability of the chance occurrence of multiple malignant neoplasms. Am J Cancer. 1934; 21(4):2309.

6. Vogt A, Schmid S, Heinimann K, Frick H, Herrmann C, Cerny $\mathrm{T}$ et al. Multiple primary tumours: challenges and approaches, a review. ESMO Open. 2017;2(2):e000172.

7. Warren S, Gates O. Multiple primary malignant tumours: A survey of the literature and statistical study. Am J Cancer. 1932; 16:1358-414.

8. Coyte A, Morrison D, McLoone P. Second primary cancer risk - the impact of applying different definitions of multiple primaries: results from a retrospective population-based cancer registry study. BMC Cancer. 2014;14(1).

9. Amer M. Multiple neoplasms, single primaries, and patient survival. Cancer Management and Research. 2014;:119.

10. Ferretti S. Airtum cancer registration handbook. Florence, Italy; 2009

11. Kim S, Kim H, Lee J, Lee Y, Kang W, Park J et al. Multiple Primary Cancers Including Colorectal Cancer. Journal of the Korean Society of Coloproctology. 2008;24(6):467.

12. Hartley A, Birch J, Kelsey A, Marsden H, Harris M, Teare M. Are germ cell tumors part of the Li-Fraumeni cancer family syndrome?. Cancer Genetics and Cytogenetics. 1989;42(2):221-226.

13. Frebourg T, Barbier N, Yan YX, Garber JE, Dreyfus M, Fraumeni J Jr., et al.Germline p53 mutations in 15 families with Li-Fraumeni syndrome. Am J Hum Genet 1995; 56:608-15.

14. Shah SA, Riaz U, Zahoor I, et al. Carcinoma multiplex. J Coll Physicians Surg Pak 2013; 23:290-2.

15. Amer M. Multiple neoplasms, single primaries, and patient survival. Cancer Management and Research. 2014;:119.

16. Weir H, Johnson C, Thompson T. The effect of multiple primary rules on population-based cancer survival. Cancer Causes \& Control. 2013;24(6):1231-1242.

17. Ricceri F, Fasanelli F, Giraudo M, Sieri S, Tumino R, Mattiello A et al. Risk of second primary malignancies in women with breast cancer: Results from the European prospective investigation into cancer and nutrition (EPIC). International Journal of Cancer. 2015;137(4):940-948.

18. Molina-Montes E, Pérez-Nevot B, Pollán M, SánchezCantalejo E, Espín J, Sánchez M. Cumulative risk of second primary contralateral breast cancer in BRCA1/BRCA2 mutation carriers with a first breast cancer: A systematic review and meta-analysis. The Breast. 2014;23(6):721-742.

19. Wallis C, Mahar A, Choo R, Herschorn S, Kodama R, Shah $\mathrm{P}$ et al. Second malignancies after radiotherapy for prostate cancer: systematic review and meta-analysis. BMJ. 2016;:i851.

20. Friedenson B. BRCA1 and BRCA2 pathways and the risk of cancers other than breast or ovarian. MedGenMed 2005; $7: 60$.

21. Bhuller K, Zhang Y, Li D, Sehn L, Goddard K, McBride $\mathrm{M}$ et al. Late mortality, secondary malignancy and hospitalisation in teenage and young adult survivors of Hodgkin lymphoma: report of the Childhood/Adolescent/ Young Adult Cancer Survivors Research Program and the BC Cancer Agency Centre for Lymphoid Cancer. British Journal of Haematology. 2016;172(5):757-768.

22. Bhaskarla A, Tang P, Mashtare T, Nwogu C, Demmy T, 
Adjei A et al. Analysis of Second Primary Lung Cancers in the SEER Database. Journal of Surgical Research. 2010;162(1):1-6.

23. Hewitt M, Greenfield S, Stovall E, editors. From cancer patient to cancer survivor: lost in transition. Washington, DC: The National Academies Press; 2006.

24. Demark-Wahnefried W, Pinto B, Gritz E. Promoting Health and Physical Function Among Cancer Survivors: Potential for Prevention and Questions That Remain. Journal of Clinical Oncology. 2006;24(32):5125-5131.

This work is licensed under a Creative Commons AttributionNon Commercial 4.0 International License. 